\title{
CHANGES IN MEDIAL CORTICAL BLOOD FLOW WITH A STIMULUS-RESPONSE COMPATIBILITY TASK
}

\author{
Stephan F. Taylor, ${ }^{*}+$ Sylvan Kornblum,,$+\ddagger$ Satoshi Minoshima,$\S$ \\ LINDSAY M. OLIVER $\ddagger$ and ROBERT A. KOEPPE $§$ \\ $\dagger$ Departments of Psychiatry, ${ }_{\ddagger}$ Psychology, and Internal Medicine, Division of Nuclear Medicine, University \\ of Michigan Medical Center, Ann Arbor, MI. U.S.A.
}

(Received 23 March 1993; accepted 28 June 1993)

\begin{abstract}
Previous work has suggested that human subjects engaged in tasks, like the Stroop task, that require response selection utilize the medial frontal cortex. We used positron emission tomography to measure blood flow changes in a stimulus-response compatibility task designed to maximize the demand on response selection processes. We report significant activation in the cingulate sulcus (Brodman's area 32) and a correlation of activity in this region with faster response time for an incongruent stimulus-response task.
\end{abstract}

\section{INTRODUCTION}

THE Stroop task consists of having people name the color in which color-words are written when the color-word and the ink color sometimes match (e.g. BLUE written in blue ink) and sometimes conflict (e.g. RED written in blue ink). Robust performance differences, such as longer reaction times and more errors on conflicting trials, have been obtained between these two conditions, making the Stroop task an important experimental paradigm for the study of selective attention and sensory-motor processes (see MACLEOD [14] for a recent review).

PARDo et al. [20] have recently reported the results of a positron emission tomography (PET) study in which subjects performing a Stroop task showed an increase in cerebral blood flow $(\mathrm{CBF})$ in several regions of the medial frontal cortex: the anterior cingulate gyrus, the anterior inferior cingulate sulcus and the supplementary motor area (SMA). Pardo et al. proposed that the medial frontal cortex, specifically the anterior cingulate gyrus, may be one of the cortical areas involved in "attention for action," what we shall refer to as the "response selection process." Their conclusion receives support from several other lines of evidence. Lesions that include this region can cause akinesia, without evidence of limb weakness [12], or transcortical motor aphasia $[1,23]$, which results in impaired spontaneous speech with a preserved ability to repeat words and phrases. During electroencephalographic recordings of subjects making voluntary, self-initiated movements, the initial component of a negative readiness potential occurring prior to the onset of self-initiated movement attains maximum change in the precentral midline $[7,11]$.

Two points can be made about the design employed by Pardo et al.: one derives from the Stroop paradigm itself, the other concerns the constraints that the PET procedure imposes

\footnotetext{
*Address for correspondence: University Hospital 8D, 1500 E. Medical Center Drive, Ann Arbor, MI 48109-0116, U.S.A.
} 
on the experimental design. The first point concerns the source of the Stroop interference. For example, consider the case where the word "RF.D" is printed in blue ink, and where the correct response consists of saying "blue." We have already noted that the reaction time for saying "blue" on this type of trial is longer than on trials where the word "BLUE" is printed in blue ink. This difference in performance has been variously attributed to a stimulus-response ( $\mathrm{S}-\mathrm{R}$ ) conflict between the word "RED" and the response "blue," and/or a stimulus-stimulus $(\mathrm{S}-\mathrm{S})$ conflict between the word "RED" and the color of the ink (which in our example is blue). Because both conflicts are actually present in such trials, it has not been possible to use these data to distinguish between the S-R and the S-S factor as the source of the performance difference. Pardo et al. are aware of this confounding, and acknowledge that their activation findings may in fact reflect these two different sources of conflict.

The second issue concerns the modifications that need to be made to the Stroop task in order to make it an appropriate behavioral protocol for use with PET scanning. During the normal administration of the Stroop task, matching and conflicting trials are randomly interspersed in a block. This produces trial-by-trial uncertainty about the relation between the color and the color-word in Stroop stimuli. However, because the PET procedure requires homogeneous conditions during the 40-60 sec of the scan, Pardo et al. had to present the matching and the conflicting trials in separate blocks, thus removing this source of uncertainty and making color and color-word redundant aspects of the stimulus. Although subjects were instructed to regard the color-words as irrelevant to the task at hand, this color/color-word redundancy could easily have permitted subjects to read the "irrelevant" color-words, rather than actually naming the ink color of the letters. Performance in the matching and in the conflicting blocks could, therefore, have been based on entirely different processes (reading versus color-naming).

KORNBLUM and his colleagues $[9,10]$ have recently proposed a model - the dimensional overlap (D.O.) model and a taxonomy in which they identify a broad array of stimulus-stimulus and stimulus-response compatibility tasks and show how these tasks are logically related to one another. In particular, they show how the Stroop task is explicitly related to other, simpler performance paradigms. Their analysis permits the two sources of conflict in the Stroop task to be unconfounded, and examined separately. In the present study we chose to isolate and to examine the stimulus-response conflict, when the conflict is with a relevant rather than with an irrelevant stimulus attribute.

The stimuli consisted of four visually-presented letters $(B, J, Q, Y)$. The responses consisted of these same letter names ("b", "j", "q", "y"). There were two conditions in the experiment: the control, or congruent, condition in which subjects responded to each letter with its own name, and the activation, or incongruent, condition in which subjects responded to each letter with the name of the other letters ("q" for B, "y" for J, "j" for Q, and "b" for Y). Because the incongruent condition required the resolution of a response conflict, we predicted, on the basis of Pardo's data and the clinical and experimental observations cited above, that such a task would activate the medial frontal cortex.

\section{METHODS}

Subjects

The subjects were cight male voluntcers, judged to be right-handed by the Edinbuigh inventory [19]. They were without past or current history of chronic medical illness, neurological illness, head injury, learning disability or psychiat ric illness. All subjects gave informed consent to participate in the study after the experimental procedure and radiation risks were explained, as per protocols approved by our local Institutional Review Board. 


\section{Behavioral task}

An experimental session lasted about $2.5 \mathrm{hr}$ and began with five practice blocks of the incongruent task, each consisting of 64 trials. A trial began with a visual warning signal followed, after $500 \mathrm{msec}$, by one of the four letters. subtending a visual angle of $1.2^{\circ}$. The subject then had to make his response as quickly, and as accurately, as he could. Following an interval of between 400 and $700 \mathrm{msec}$ after the response, the warning signal for the next trial was presented. Subjects could earn a bonus based on a performance score they had accumulated. Four to six scans were done on each subject-half with the congruent, half with the incongruent condition. These conditions were alternated and balanced over subjects. Scans were made 14 minutes apart. Between scans, subjects performed two biocks on the condition that would be used in the next scan. The hehavioral task began approximately 30 sec prior to the start of the scan.

\section{PET Scanning}

The subjects underwent PET scans in a Siemens CTI 931/08-12 camera (CTI Inc, Knoxville, TN) which acquires 15 slices over an axial length of $9.5 \mathrm{~cm}$. Before emission scanning, subjects had attenuation measured by an external ${ }^{68} \mathrm{Ge} /{ }^{68} \mathrm{Ga}$ ring source. For each emission scan, subjects were given an i.v. bolus injection of $50-66 \mathrm{mCi}$ of $[\mathrm{O}-15] \mathrm{H}_{2} \mathrm{O}$. Data were collected in a single $60 \mathrm{sec}$ frame beginning $5 \mathrm{sec}$ after the arrival of the radioactivity in the brain. Scans were reconstructed with a Parzen filter to yield an effective full width at half-maximum (FWHM) of 9-10 mm.

We proportionally normalized tracer counts to the global mean, arbitrarily set to 1000 , and performed the analysis on relative tissue activity. Since relative activity bears a linear relationship with measurements of relative $\mathrm{CBF}$, this technique avoids the use of an arterial catheter [4]. We used automated programs developed and validated in our lahoratory $[15,16]$ to re-orient, re-slice and transform the image arrays into stereotactic space [25]. To remove individual differences in gyral anatomy, these images were further smoothed with a three-dimensional Gaussian filter to give an effective resolution of approximately $14 \mathrm{~mm}$ (FWHM). Differences between the control and activation images, each initially averaged within subjects, were expressed as voxel-by-voxel $t$-scores and then normalized into $z$-scores [5]. In order to calculate the significance threshold for the resulting $z$-image, we used a three-dimensional modification of a method described by FrisTON et al. [5], which sets a significance threshold after adjusting for multiple comparisons and the inherent non-independence of neighboring pixels.

\section{RESULTS}

The behavioral results are quite clear: mean reaction time in the congruent mapping condition was $455 \mathrm{msec}$ (S.D. \pm 61 ), and for the incongruent mapping condition $680 \mathrm{msec}$ (S.D. \pm 77$)$, thus yielding a mapping effect of $224 \mathrm{msec}(t=15.2$, d.f. $=7, P<0.0001)$.

Within our hypothesized area of interest, the medial frontal cortex, the cingulate sulcus, at Brodman's area (BA) 32 , was the only region in which activation exceeded the $z$-value cutoff threshold of 3.7 (see Fig. 1 and Table 1). The activation focus lateralized to the left cingulate sulcus, although there was non-significant activation occurring to the right of the midline. Observations of the individual responses demonstrated that the activation tended to occur bilaterally, although more consistently on the left, thereby causing the $z$-score to peak $10 \mathrm{~mm}$ off the midline. Two other significant peaks of activation occurred, on the right and left inferior frontal gyrus (BA 47).

For each subject, the 2 or 3 images acquired during each condition were averaged in stereotactic space. A spherical region of interest $(20.25 \mathrm{~mm}$ diameter $)$ was centered at the stereotactic coordinates of the mean activity of the cingulate sulcus peak and relative counts were measured for each subject for each condition. We found a significant Pearson product moment between mean reaction time (RT) in the incongruent condition and relative activity at the cingulate sulcus during that condition (Fig. $2 ; r=0.77$, d.f. $=7, P=0.03$ ); however, this calculation failed to reach significance for the congruent condition $(r=0.43$, d.f. $=7$, n.s. $)$.

\section{DISCUSSION}

Although the congruent condition in Pardo et al. and in our own study are similar, in that they both involve the reading of words or letters, the incongruent conditions are quite 


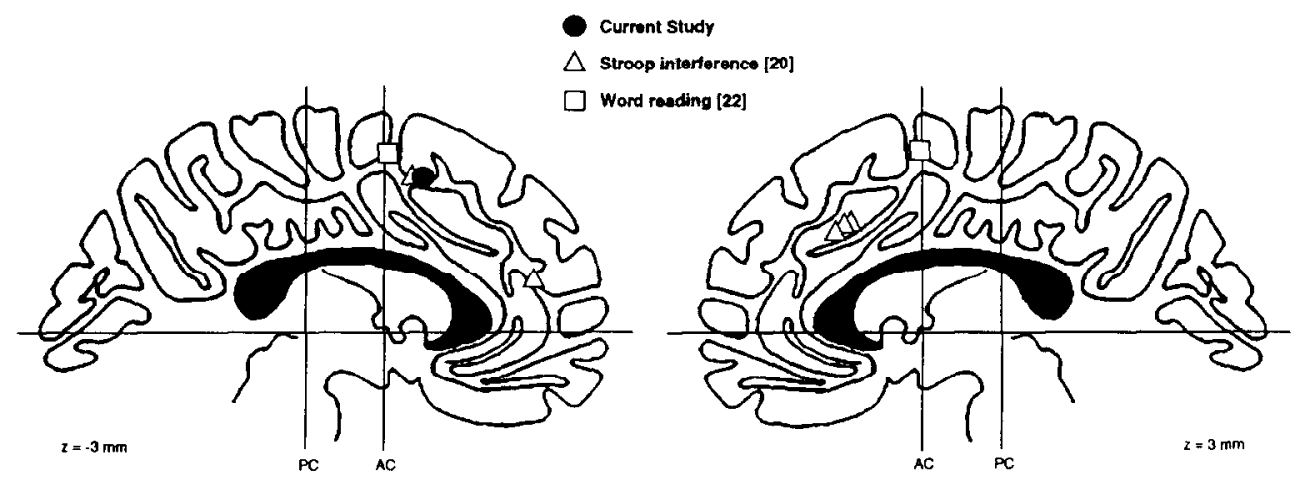

Fig. 1. The figure compares activation peaks in the medial frontal cortex from published studies with the peak obtained from the 21 scan pairs in eight subjects in the current study. Vertical lines pass through the anterior commissure $(\mathrm{AC})$ and posterior commissure $(\mathrm{PC})$; the horizontal line passes through both and defines the $0,0,0$ point of the stereotactic coordinate system at the anterior commissure. The sagittal sections from the reference atlas [25] are located $3 \mathrm{~mm}$ from either side of the midline.

Table 1. Location of activation foci

\begin{tabular}{lrc}
\hline Region & $\begin{array}{c}\text { Coordinates* } \\
(x, y, z)\end{array}$ & $z$-score $\dagger$ \\
\hline L cingulate sulcus (BA 32) & $10,14,43$ & 5.5 \\
R inf. frontal gyrus (BA 47) & $-37,17,-2$ & 8.2 \\
L inf. frontal gyrus (BA 47) & $39,17, \quad 2$ & 4.3 \\
\hline
\end{tabular}

*Coordinates from the atlas of TAlairach and Tournoux [25], where " $x$, " $y$ " and ' $z$ ' correspond to the right-left, anterior-posterior and superior-inferior dimensions, respectively.

$\downarrow z$-score of peak pixel, derived from a normalized $t$-statistic calculated on a voxel-by-voxel basis.

different, for in our study there were no dimensional conflicts in the stimuli. The only conflict that occurred in our study was in the incongruent condition, and that conflict was between the relevant stimulus and the required response. That is, when presented with the letter $\mathrm{B}$, for example, subjects were required to suppress the automatically-activated name of the letter " $b$ " and produce the name of another letter in the set, say "q". This is analogous to reading the irrelevant word "RED" in a conflicting Stroop trial and suppressing it in order to produce the correct response "blue", which is the color of the ink (see KornbLum [9] for a more detailed exposition of the processes involved).

We found activation in the cingulate sulcus, very close to a region that Pardo et al. identified as SMA (Fig. 1). Both of these responses lateralized to the left hemisphere, which may reflect their involvement in language output. However, we did not find an activation peak in the anterior cingulate region, which Pardo et al. identified as being responsible for response selection. We interpret these findings as suggesting that the cingulate sulcus/SMA region is involved in resolving the response conflicts in both our simple stimulus-response compatibility task and in the Stroop task. The strong correlation of the cingulate sulcus activity with response time in the incongruent condition provides additional evidence 


\section{Incongruent Mapping Condition}

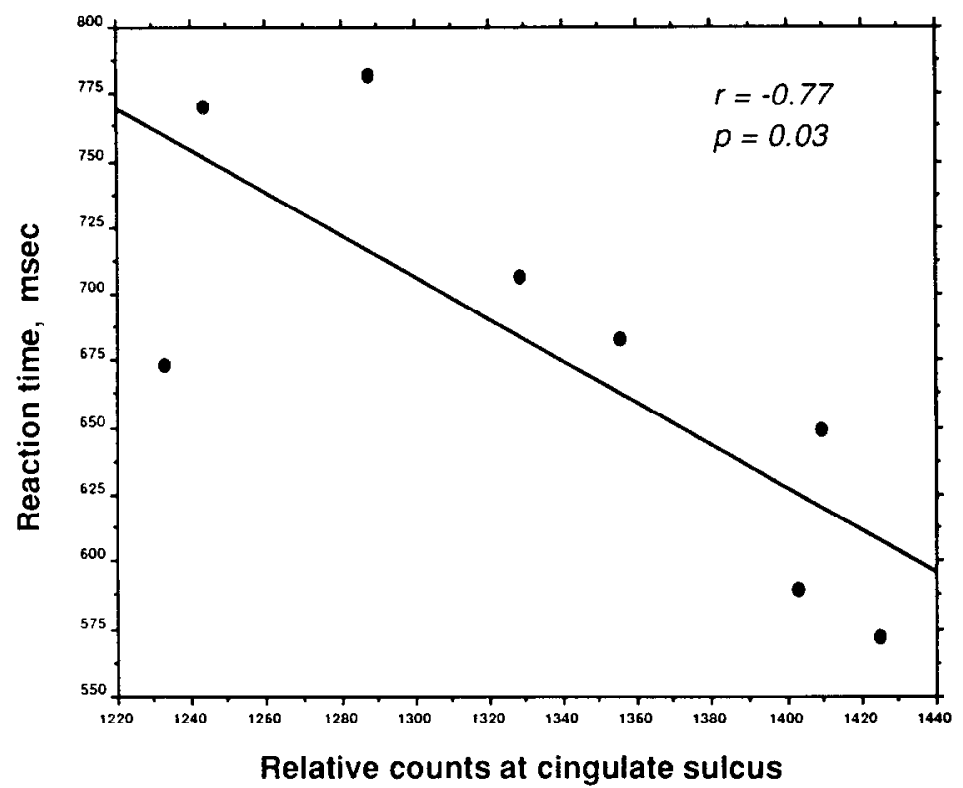

Fig. 2. The graph plots the significant relationship between each subject's mean reaction time during the incongruent condition and the activity of the cingulate sulcus during this condition. Subjects who responded faster had less activity in this region. This relationship was not significant for the congruent condition.

consistent with the role of this region in resolving the response conflict, i.e. response selection. On the other hand, the anterior cingulate region, absent in our findings, may be involved in the resolution of stimulus-stimulus conflicts requiring selective attention. A role for this anterior cingulate region in the selection of stimuli for processing is supported by other PET activation studies $[3,6]$.

The involvement of the cingulate sulcus in motor processing was suggested by the Pardo $e t$ al. study when they identified the region as SMA. However, in a task where subjects read words aloud, PETERSON et al. [22] found SMA activation $1 \mathrm{~cm}$ superior and posterior to this site. We have also studied subjects in this same reading task, and we also found this SMA site in a more superior and posterior location than our cingulate sulcus site (TAYLOR et al., unpublished data). This cingulate sulcus site may represent a motor area distinct from the traditional SMA. Studies in primates have also suggested the existence of a somatotopic supplementary motor region in the ventral bank of the cingulate sulcus, distinct from the traditionally-defined SMA $[13,18]$. Although the technique of image averaging can resolve activation peaks at a resolution less than the FWHM of the reconstructed image [17], one should exercise caution while comparing these areas, especially between laboratories. Further work is clearly needed to make additional functional delineation between the SMA and the cingulate sulcus region in humans.

We also activated the left and right inferior frontal gyrus (BA 47), which was not predicted on the basis of the Stroop study. One possibility is that these regions performed mnemonic functions, not present in the Stroop task, but required by our study. The activation on the left 
inferior frontal gyrus was close to a region activated by working memory studies designed to utilize a "phonological loop" [21] (KOEPPE et al., unpublished data). Many of our subjects reported using phonological associations to a word as a mnemonic key, e.g. "barbecue" for the " $\mathrm{B}$ " to " $\mathrm{Q}$ " mapping. In a working memory study by JoNiDEs et al. [8], subjects activated the right $\mathrm{BA} 47$ for a task requiring the retention of a spatial configuration in working memory. Since it is difficult to argue that our subjects used a "spatial strategy" for our task, an alternative interpretation might be that the inferior frontal gyrus activations represent the activity of a "central executive" or a "supervisory attentional system" $[2,24]$ which allocate resources for the performance of this task, as well as the working memory tasks.

The current study provides some new information about the functional role of regional brain activity involved in the resolution of Stroop-like conflicts. While the Pardo study activated several other regions that we did not activate, such as peristriate cortex, pre- and post-central sulcus and superior temporal gyrus, these differential activations probably say more about the extent to which the Stroop task and our task differed than anything else. A true functional neuroanatomy emerges through this sort of iterative process, whereby experimental results in converging and diverging paradigms are predicted by and modify our theoretical constructs.

Acknowledgements-The authors would like to acknowledge Bernard Agranoff for advice and support, Anthony Whipple for technical assistance and data analysis, and JoAnn Goodson for testing the subjects. This work was supported by a grant from the University of Michigan GCRC (M01-RR00042). SK received partial support from the National Institute of Mental Health (R01-MH43287), Office of Naval Research (ON00014-89-J-1557) and NATO (860215).

Part of the work has been presented at the XVIth International Symposium on Cerebral Blood Flow and Metabolism, Sendai, Japan, May 1993.

\section{REFERENCES}

1. Alexander, M. P. and Schmitt, M. A. The aphasia syndrome of stroke in the left anterior cerebral artery territory. Ann. Neurol. 37, 97-100, 1980.

2. Baddeley, A. D. Working Memory: Oxford University Press, Oxford, 1986.

3. Corbetta, M., Miezin, F. M., Shllman, G. L. and Petersen, S. E. Selective attention modulates extrastriate visual recognition regions in humans during visuat feature discrimination and recognition. In Exploring Brain Functional Anatomy with Positron Tomography, D. J. CHADwick and J. WHELAN (Editors), pp. 165-175. John Wiley \& Sons, London, 1991

4. Fox, P. T. and Mintlix, M. A. Noninvasive functional brain mapping by change-distribution analysis of averaged PET images of $\mathrm{H}_{2}{ }^{15} \mathrm{O}$ tissue activity. J. Nucl. Med. 30, $141-149,1989$.

5. Fristox, K, J., Frith, C. D., Liddle, P. F. and Frackowiak, R. S. Comparing functional (PET) images: the assessment of significant change. J. Cereb. Blood Flow Metab. 11, 690-699, 1991

6. Friston, K. J., Frith, C. D., Liddle, P. F. and Frackowlak, R. S. J. Functional connectivity: The principalcomponent analysis of large (PET) data sets. J. Cereb. Blood Flow Metab. 13, 5 14, 1993.

7. Grozinger, B., Kornhlber, H. H. and Kriebel, J. Participation of mesial cortex in speech: evidence from cerebral potentials preceding speech production in man. In Hearing Mechanisms and Speech, O. CREUTZFELD, H. SCHEICH and C. SChreiner (Editors), pp. 189-192. Springer, Berlin, 1979.

8. Jonides, J., Smith, E. E., Koeppe, R. A., Awh, E., Minoshima, S. and Mintun, M. Spatial working memory in humans as revealed by PFT. Nature 363, 623-625

9. Kornblem, S. Dimensional overlap and dimensional relevance in stimulus response and stimulus-stimulus compatibility. In Tutorials in Motor Behatior II. G. E. StelmaCH and J. ReQuiN (Editors), pp. 743-777. Elsevier, Amsterdam, 1992.

10. Kornblem, S.. Hasbrolce, T. and Osman, A. Dimensional overlap: Cognitive basis for stimulus-response compatibility-A model and taxonomy. Psychol. Rer. 97, 253-270, 1990

11. Lang, W., Cheyne, D., Kristeva, R., Beistfiner, R., Lindinger, G. and Deeck, L. Three-dimensional localization of SMA activity preceding voluntary movement. Exp. Brain Res. 87, 688-695, 1991.

12. Laplane, D., Talairach, J., Meininger, V., Bancald. J. and Orgogozo, J. M. Clinical consequences of corticectomies involving the supplementary motor area in man. J. Neurol. Sci. 34, 301-314, 1977.

13. Llppino, G., Matelli, M., Camarida, R. M.. Gallese. V. and Rizzolatti, G. Multiple representations of 
body movements in mesial area 6 and the adjacent cingulate cortex: An intracortical microstimulation study in the macaque monkey. J. Comp. Neurol. 311, 463-482, 1991.

14. MacLeod, C. M. Half a century of research on the Stroop Effect: An intergrative review. Psychol. Bull. 109, $163-203,1991$.

15. Minoshima, S., Berger, K. L., LeE, K. S. and Mintle, M. A. An automated method for rotational correction and centering of three-dimensional functional brain images. $J$. Nucl. Med. 33, 1579-1585, 1992.

16. Minoshima, S., Koeppe, R. A., Mintle, M. A., Berger, K., Taylor, S. F., Frey, K. A. and Kuhl, D. E. Automated detection of the intercommissural $(\mathrm{AC}-\mathrm{PC})$ line for stereotactic localization of functional brain images. J. Nucl. Med. 34, 322-329, 1993.

17. Mintun, M. A., Fox, P. T. and Raichle, M. E. A highly accurate method of localizing regions of neuronal activation in the human brain with positron emission tomography.J. Cereb. Blood Flow Metab. 9, 96-103, 1989.

18. Muakkassa, K. F. and Strick, P. F. Frontal lobe inputs to primate motor cortex: Evidence for four somatotopically organized 'premotor' areas. Brain Re's. 177, 176-182, 1979.

19. Oldfield, R. C. The assessment and analysis of handedness; the Edinburgh inventory. Neuropsychologia 9 , 97. $113,1971$.

20. Pardo, J. V., Pardo, P. J., J Aner, K. W. and Raichle, M. E. The anterior cingulate cortex mediates processing selection in the Stroop attentional conflict paradigm. Proc. Natl. Acad. Sci. U.S.A. 87, 256-259, 1990.

21. Pallest, E., Frith, C. D. and Frackowiak, R. S. J. The neural correlates of the verbal component of working memory. Nature, 362, $342 \cdot 344,1993$.

22. Petersen, S. E., Fox, P. T., Posner, M. I., Mintun, M. and Raichle, M. E. Positron emission tomographic studies of the cortical anatomy of single-word processing. Nature 331, 585-589, 1988.

23. RubENS, A. B. Aphasia with infarction in the territory of the anterior cerebral artery. Cortex 11, 239-250, 1975.

24. Shallice, T. From Neuropsychology to Mental Strucrure. Cambridge University Press, New York, 1988.

25. Talairach, J. and Tolrnoux, P. A Co-planar Stereotaxic Atlas of a Human Brain. Thieme, Stuttgart, 1988. 